\title{
Cystite gangréneuse de la vessie compliquée d'une perforation spontanée en intrapéritonéal
}

\author{
Mohamed Ali Ben Chehida, MD; Mohamed Chlif, MD; Ahmed Saadi, MD; Mourad Mohamed Gargouri, MD; \\ Ahmed Sellami, MD; Sami Ben Rhouma, MD; Yassine Nouira, MD
}

Service d'urologie, Hôpital La Rabta de Tunis, Tunisie

Cite as: Can Urol Assoc J 2016;10(7-8):E270-3. http://dx.doi.org/10.5489/cuaj.3147 Published online July 12, 2016

\section{Résumé}

La cystite gangréneuse compliquée d'une perforation en intrapéritonéal est une pathologie extrêmement rare. Le facteur étiopathogénique principal est l'ischémie de la paroi vésicale. L'intoxication alcoolique comme facteur à l'origine d'une ischémie pariétale constitue un mécanisme complexe mais grave du fait de l'étendue des lésions et du terrain sur lequel se greffe cette complication. Ce cas clinique rapporte l'observation d'un patient de 36 ans, alcoolique chronique, hospitalisé en raison d'un tableau clinique de péritonite aiguë; L'exploration chirurgicale a révélé une gangrène localisée de la vessie à l'origine d'une perforation spontanée en intrapéritonéal. Après réparation chirurgicale, la fonction et la capacité vésicales sont revenues à la normale.

\section{Introduction}

La cystite gangréneuse est une pathologie définie par la nécrose de la muqueuse et de la sous-muqueuse vésicales. La nécrose peut atteindre la totalité de la paroi vésicale, incluant la musculeuse et la séreuse, conduisant ainsi à une rupture spontanée dans la cavité péritonéale ${ }^{1}$.

La cystite gangréneuse est une pathologie rare mais connue depuis le $17^{\mathrm{e}}$ siècle ${ }^{2}$. Elle a disparu depuis l'avènement de I'antibiothérapie et le développement des moyens de drainage vésical. Néanmoins, quelques cas rares ont été décrits récemment dans la littérature ${ }^{3}$.

Nous rapportons un cas de cystite gangréneuse compliquée $d^{\prime}$ une perforation spontanée de la vessie en intrapéritonéal.

\section{Cas clinique}

Il s'agit d'un jeune homme de 36 ans, alcoolique chronique au stade $d^{\prime}$ insuffisance hépatique, admis aux urgences pour douleurs abdominales diffuses avec notion d'hématurie totale évoluant depuis une semaine.
À l'examen, le patient n'était pas fébrile mais son état général était très altéré avec un tableau clinique évoquant une insuffisance rénale aiguë. L'abdomen était très distendu, douloureux et en contracture à la palpation, ce qui a conduit à un diagnostic de péritonite aiguë.

Un scanneur en urgence a révélé un important épanchement intrapéritonéal en rapport avec une brèche vésicale au niveau de sa face postérieure (fig. 1).

Les analyses de laboratoire étaient compatibles avec un syndrome inflammatoire biologique. La CRP était élevée et accompagnée d'une leucocytose $\left(26000 / \mathrm{mm}^{3}\right)$ et d'une insuffisance rénale aiguë avec une créatinémie à $86 \mathrm{mg} / \mathrm{L}$ associée à des troubles ioniques majeurs ayant nécessité le recours à l'hémodialyse.

Après réanimation, le patient a été opéré d'urgence. La laparotomie d'exploration a révélé un épanchement intrapéritonéal de plus de 2 litres de liquide trouble et sérosanguin. La vessie était le siège d'une perforation d'environ $4 \mathrm{~cm}$ de diamètre avec des berges nécrotiques situées au bas fond vésical; I'exploration de la vessie a révélé une forte inflammation de la muqueuse mais sans lésion tumorale. Le reste de la cavité vésicale était indemne (fig. 2A, 2B).

Une excision des berges de la perforation a été effectuée jusqu'au tissu sain. Le tissu excisé a été envoyé pour examen anatomopathologique en extemporané et l'analyse a conclu à la présence de lésions de cystite gangréneuse sans signe de malignité (fig. 3).

La perforation a été par la suite suturée en deux plans et protégée par une sonde de cystostomie et une sonde transurétrale. La sonde de cystostomie a été retirée au $6^{\mathrm{e}}$ jour post opératoire. Le drainage par la sonde vésicale a été prolongé pendant 15 jours et associé à une biantibiothérapie; aucune complication postopératoire n'a été observée. La fonction rénale s'est normalisée avec reprise d'une miction normale. L'intégrité de la vessie a été vérifiée cliniquement et radiologiquement par cystographie jusqu'à 14 mois en postopératoire sans qu'aucune fuite ne soit notée. 


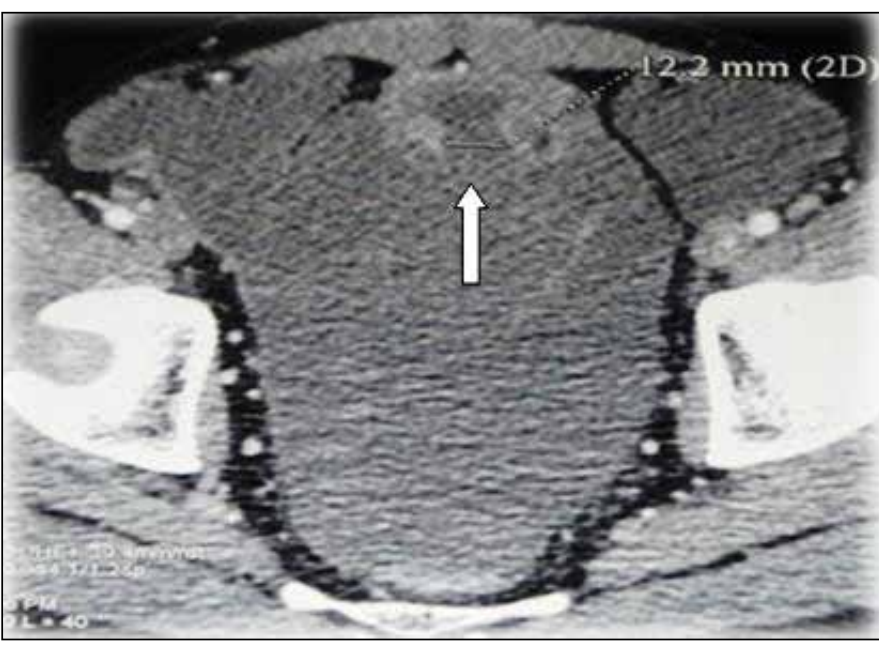

Fig. 1. Brèche vésicale au niveau de la face antérieure de la vessie (flèche blanche) associée à un épanchement intrapéritonéal.

\section{Discussion}

Le premier cas de cystite gangréneuse de la vessie a été rapporté par Willis en $1650^{1,2}$. C'est une entité rare et seulement un total de 240 cas dans le monde ont été signalés jusqu'à présent ${ }^{4}$. Sur le plan épidémiologique, cette entité se distinguait par une nette prédominance féminine, attribuée à la fréquence des traumatismes obstétricaux au cours du travail et l'accouchement. Le pronostic était très sombre, le taux de mortalité avoisinant $60 \%$ et le diagnostic se faisait souvent au cours des autopsies ${ }^{1,3,5,6}$.

À l'heure actuelle, la cystite gangréneuse est devenue une complication exceptionnelle, voire historique, rarement rapportée dans la littérature ; elle a subi aussi une inversion du ratio entre les sexes et une diminution du taux de mortalité, qui s'établit maintenant à $32 \%{ }^{3,4,5}$.
L'étiologie de la cystite gangréneuse consiste en une ischémie de la paroi vésicale favorisée par des antécédents chirurgicaux ou médicaux qui agissent soit directement, comme une irradiation pelvienne ou une irritation chimique, la plupart du temps d'origine médicamenteuse (cyclophosphamides), soit indirectement en privant la vessie de ses apports vasculaires et nutritifs, tels lors d'une chirurgie pelvienne antérieure, une distension vésicale chronique ou une athérosclérose des vaisseaux du petit bassin ${ }^{1,4}$.

Cette ischémie pariétale fragilise la paroi vésicale, qui devient vulnérable à la moindre surdistension, laquelle provoque alors la perforation ${ }^{1,4}$.

L'infection bactérienne comme cause directe de gangrène vésicale a presque disparu avec l'arrivée de l'antibiothérapie, mais sa participation secondaire après une ischémie pariétale par distension vésicale chronique est presque constante. Les germes incriminés sont soient aérobies (Proteus, staphylocoques, E. Coli) ou anaérobies (streptocoques, Clostridium, Bacteroides) ${ }^{1,2}$.

Dans notre cas, l'étiologie répond aux mêmes principes déjà décrits mais avec un facteur supplémentaire : I'alcoolisme chronique. Son association avec la gangrène vésicale a été décrite ${ }^{7}$. L'intoxication alcoolique provoque une polyurie compliquée d'une dysectasie au niveau du col vésical et une insensibilité au besoin mictionnel d'origine neurologique. Par ailleurs, la musculature abdominale, et notamment les muscles grands droits, sidérés par l'intoxication alcoolique, n'assurent plus leur rôle habituel de protection contre les mini-traumatismes. Il suffit alors d'un à-coup d'hyperpression faisant suite à un effort de vomissement (fréquent en cas d'éthylisme) pour que la paroi vésicale cède ${ }^{7}$.

Le siège de la perforation est souvent le même, soit au niveau du bas fond vésical. La situation anatomique de la vessie en est la cause, car les faces antérieure et latérale et

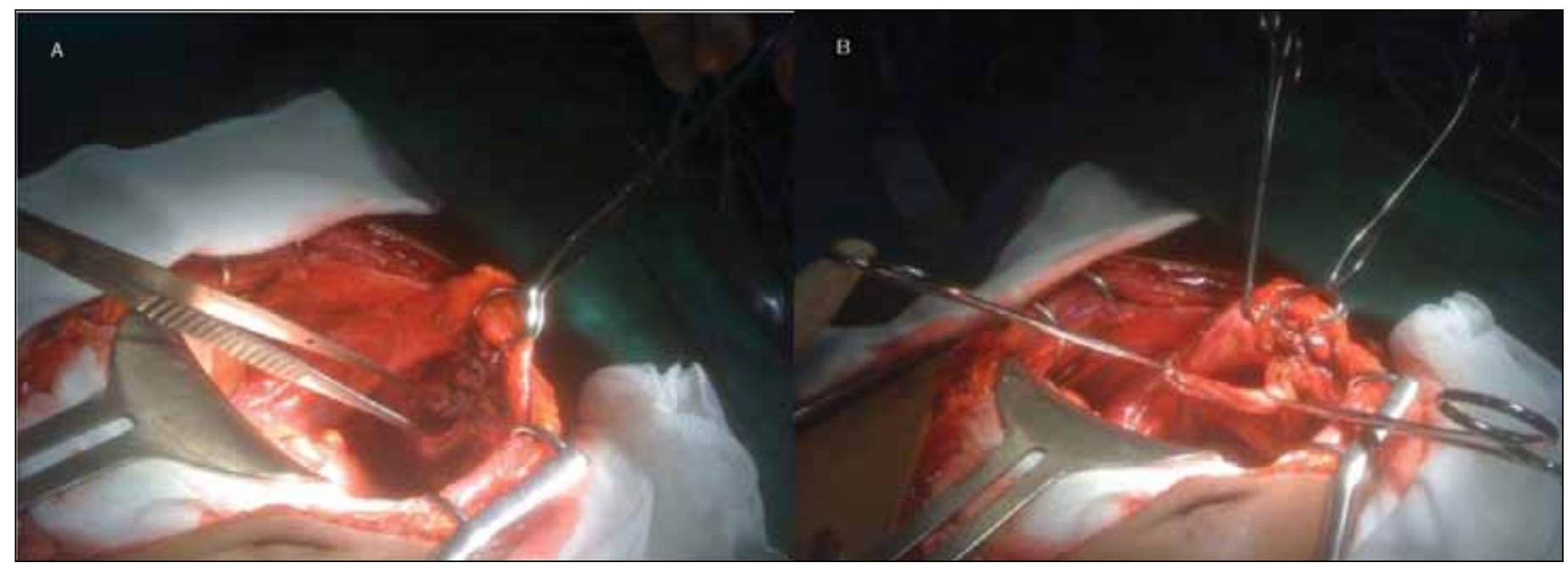

Fig. 2. (A) Perforation vésicale en intrapéritonéal; (B) Perte de substance de $4 \mathrm{~cm}$ au niveau du bas-fond vésical d'aspect nécrotique. 


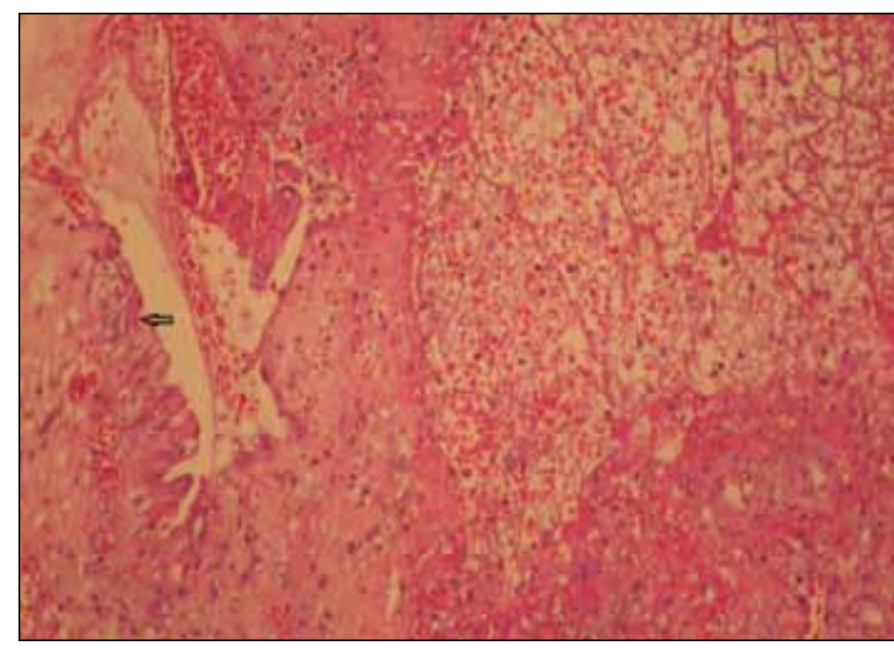

Fig. 3. Aspect histologique du tissu excisé : remaniements hémorragiques et nécrotiques (HEx40).

le trigone sont protégés par la trame musculo-osseuse du pelvis; alors que le dôme et le bas-fond sont (à vessie pleine) intrapéritonéaux. Ils subissent donc les vecteurs forces de la surdistension et par conséquent, la rupture survient au dôme ou au bas-fond vésical et se fait en intrapéritonéal ${ }^{4}$.

Le diagnostic est généralement difficile à poser à tous les stades de la maladie, aussi bien au début de l'incident qu'au cours de l'installation du tableau clinique. Les signes urinaires sont non spécifiques, évoquant une cystite simple (hématurie, impériosité mictionnelle, brûlures mictionnelles, cystalgies), mais brutalement, l'évolution se fait vers un tableau clinique de péritonite aiguëe ${ }^{4,5}$.

La gravité des signes fonctionnels et cliniques dépend étroitement de la présence ou non de surinfection bactérienne lors de la perforation, ce qui est souvent le cas, et le tableau clinique prédominant est celui de péritonite aiguë avec état de choc septique ${ }^{5}$.

Donc, devant tout tableau de péritonite précédé par une anamnèse de troubles urinaires irritatifs ou obstructifs du bas appareil et en dehors d'une notion de traumatisme, le diagnostic de perforation spontanée de la vessie doit être évoqué comme diagnostic différentiel.

Au moindre doute, un uroscanneur sensibilisé par une opacification rétrograde de la vessie est recommandé; une cystoscopie, si les conditions cliniques le permettent, peut être très utile, notamment afin d'éliminer une cause néoplasique.

Une élévation importante des valeurs de créatinine avec résolution rapide est un élément clé d'orientation diagnostique. II est expliqué par l'autodialyse transpéritonéale des urines issues de la perforation, favorisant un afflux important d'urée et de créatinine vers le contingent plasmatique suivant un gradient de concentration ${ }^{7}$.

Les particularités anatomiques de la vessie dictent la conduite thérapeutique : les pédicules vasculaires de la vessie abordent la paroi au niveau trigonal, créant ainsi une trame vasculaire en faisant respectivement des anastomoses entre eux-mêmes et les collatérales en provenance des uretères et de la prostate ou de l'utérus, selon le sexe . Le trigone, étant bien vascularisé, se trouve donc toujours épargné, quelle que soit l'étendue de la nécrose vésicale.

Ceci a un impact thérapeutique : quelle que soit l'étendue de la nécrose, le traitement doit demeurer conservateur, car en postopératoire la vessie résiduelle va augmenter de volume puisque le trigone permettra une régénération cellulaire restituant à la vessie une capacité quasi normale.

Un traitement conservateur par les antibiotiques seuls peut être envisagé théoriquement en cas de nécrose limitée à la muqueuse vésicale; ceci n'a pas été décrit dans la littérature sur le plan pratique puisque le diagnostic est souvent fait à un stade tardif $3,8,9$.

Le traitement de référence en matière de gangrène vésicale perforée est chirurgical. II consiste en une laparotomie d'exploration, un lavage péritonéal abondant et un débridement étendu des zones de nécrose avec excision des berges de la perforation pour passer en tissu sain. Habituellement, une cystectomie partielle, souvent protégée par sonde de cystostomie, suffit à enlever tout le tissu nécrosé ${ }^{4}$.

Une dérivation urinaire externe est exceptionnellement nécessaire dans les cas de nécrose totale de la vessie ${ }^{1,3}$.

Le pronostic de cette maladie est étroitement lié au terrain de la maladie. En effet, malgré un traitement chirurgical approprié, le taux de mortalité reste élevé vu les retards diagnostiques et le terrain souvent fragile et approche les $32 \%{ }^{2,4,5}$. Ce pronostic a été transformé par la large diffusion des antibiotiques ${ }^{4,5}$. Ces mêmes antibiotiques dont le spectre a évolué ont gagné en efficacité. D'autre part, les progrès de l'imagerie et de la biologie moléculaire ont contribué à mieux comprendre les mécanismes physiopathologiques de cette maladie, et donc à améliorer sa prise en charge par le biais d'un diagnostic précoce et efficace.

Intérêts conflictuels : Les auteurs affirment ne pas avoir d'intérêts conflictuels d'ordre financier ou personnel.

Cet article a été révisé par un comité de lecture.

\section{Références}

1. Deviit AT, Sethia KK. Gangrenous cystitis: Case report and review of the literature. J Urol 1993; 149:15445.

2. Maggio AJ, Andrei L. Gangrene of bladder. Urology 1981;18:390-1. http://dx.doi.org/10.1016/00904295(81)90398-8

3. Ballas K, Rafailidis S, Pavlidis T et al. Gangrenous cystitis. Int Urogynecol J Pelvic Floor Dysfunct 2007; 18:1507-9. http://dx.doi.org/10.1007/s00192-007-0430-9

4. Hinev A, Anakievski D, Krasnaliev I. Gangrenous cystitis: Report of a case and review of the literature. Urol Int 2010;85:479-81. http://dx.doi.org/10.1159/000321010 
5. De Rosa A, Amer T, Waraich N et al. Gangrenous cystitis in a 42-year-old male. BMJ Case Rep 2011 Feb 2; pii: bcrl120103526.

6. Bragança N, Cruz T, Fonseca J et al. Gangrenous cystitis. Acta Med Port 2001;14:357-9.

7. Munshi IA, Hong JJ, Mueller CM et al. Spontaneous rupture of the urinary bladder in the alcoholic patient. J Trauma 1999;46:1133-4. http://dx.doi.org/10.1097/00005373-199906000-00033

8. Gutierrez Minguez E, Arroyo Mu-0z JL, Espiga Santamaria FJ et al. Gangrenous cystitis, not such a rare pathology. Presse Med 1996;25:1390.
9. Walter S, Mogensen P, Jacobsen GK. Gangrenous cystitis: A case report. Scand J Urol Nephrol 1981; 15:73-4. http://dx.doi.org/10.3109/00365598109181217

Correspondence: Dr. Ahmed Saadi, Service d'urologie, Hôpital La Rabta de Tunis, Tunisie; saadiahmedrabta@gmail.com 